\begin{tabular}{|c|c|c|}
\hline ITC $1 / 47$ & \multicolumn{2}{|c|}{$\begin{array}{c}\text { Backward Adaptive and Quasi-Logarithmic Quantizer } \\
\text { for Sub-Band Coding of Audio }\end{array}$} \\
\hline Vol. $47 /$ No. $1 / 2018$ & Received 2017/09/06 & Accepted after revision 2018/01/12 \\
\hline $\begin{array}{l}\text { DO1 10.5755/j01.itc.47.1.16190 } \\
\text { ๑) Kaunas University of Technology }\end{array}$ & \multicolumn{2}{|c|}{ erossef http://dx.doi.org/10.5755/j01.itc.47.1.16190 } \\
\hline
\end{tabular}

\title{
Backward Adaptive and Quasi-Logarithmic Quantizer for Sub-Band Coding of Audio
}

\section{Stefan Tomić, Zoran Perić, Milan Tančić, Jelena Nikolić}

University of Niš, Department of Telecommunications, Aleksandra Medvedeva 14, 18000 Niš, Serbia e-mails: stefan@elfak.rs, zoran.peric@elfak.ni.ac.rs, milan_tancic@yahoo.com, jelena.nikolic@elfak.ni.ac.rs

Corresponding author: stefan@elfak.rs

This research presents an audio coding scheme, based on sub-band coding (SBC), with the implementation of quasi-logarithmic compandors. The presented coding scheme is based on signal decomposition and individual processing of the different sub-bands. Two SBC schemes for audio coding are presented, a non-adaptive and an adaptive coding scheme. The application of backward adaptation technique further improves the performance of this coding scheme, especially when using smaller compression factor values. This paper also describes the determination of an efficient bit allocation, used for coding the individual sub-bands. The results indicate that the proposed coding schemes can successfully be implemented in audio signal coding, providing a high quality output signal.

KEYWORDS: quantization, sub-band coding, audio signals.

\section{Introduction}

Audio (speech or music) signal carries information that is important to people. Many of the popular systems and applications that surround us are based on the latest audio technologies. This gives great significance to the research in the field of digital signal processing. Digital signal processing includes signal coding and compression, for the purpose of reducing the amount of resources required for storing a digital representation of the signal [6]. Moreover, a compressed signal can be transmitted more efficiently by using fewer resources.

Sub-band coding has been widely implemented in audio, still images and video coding [4, 12, 15-16]. This technique represents a form of transform coding, 
which applies certain transformations to the input signal, for achieving more efficient data compression. In the frequency domain, these transformations represent the application of finite impulse response (FIR) digital filters. These filters perform decomposition of the input signal into sub-bands (spectral components). As the input signal is often characterized by unequal distribution of spectral energy, it is suitable to apply the SBC scheme in order to threat the sub-bands independently [6]. SBC process typically starts by passing the input signal through the time and frequency mapping, which decomposes the signal into multiple frequency sub-bands [6]. Each of the sub-bands has specific statistical and perceptual characteristics so that the different coding schemes and bit allocations can be applied to each individual sub-band. Unlike the coding schemes presented in [15] and [16], the coding scheme we design herein implements simple transformations and quantization techniques. This outputs to relative low overall computational complexity and simple coder realization.

In [3], a speech signal coding scheme based on subband coding has been presented. It performs decomposition of the input into low and high frequency domain. Speech signal sub-band coding has also been presented in [9], with the implementation of digital signal processing. While both papers present subband coding schemes, they do not make emphasis on the quantization and do not consider evaluating objective quality of the output signal through an objective quality measure. In contrast, we present two audio coding schemes based on sub-band coding with the objective performance analysis and experiments on real input audio signals.

Most signals with quasi-static nature have in com- mon that their variance and autocorrelation function changes slowly in time [6]. Analogue to digital (A/D) convertors are often designed with the assumption that the signal source is stationary. Efficiency and characteristics of a convertor can be significantly improved by applying an adaptation on low variable statistics of the input signal. Adaptation is usually performed as adaptive quantization with

forward adaptation (AQF) and adaptive quantization with backward adaptation (AQB) [6]. Different variations of adaptation technique can be found in literature, such as switched-adaptive quantization technique, presented in [11]. This technique performs adaptation in the choice of most suitable quantizer for the statistics of the input signal. In this paper, an adaptive quantization with backward adaptation is applied. Unlike forward adaptation, backward adaptation does not require usage and transmission of the side information, as the adaptation is performed by using already transmitted samples. Reuse of the already processed samples does not increase the bit rate used for coding and does not significantly increase the computational complexity of the coding scheme.

Audio signal can have unpredictable statistical characteristics, so we assume it is the most appropriate to use logarithmic quantizers, which provide robustness in a wide range of input signal variances. In particular, our SBC schemes implement a non-adaptive and adaptive form of quasi-logarithmic quantization. Quasi-logarithmic quantizer represents a logarithmic quantizer defined with the $\mu$ compression law. This type of quantizer is implemented in defining the G.711 coding standard, according to which speech signal is coded by using 8 bit per sample with the implementation of Pulse Code Modulation (PCM) [5-6].

\section{Figure 1}

The proposed SBC coding scheme

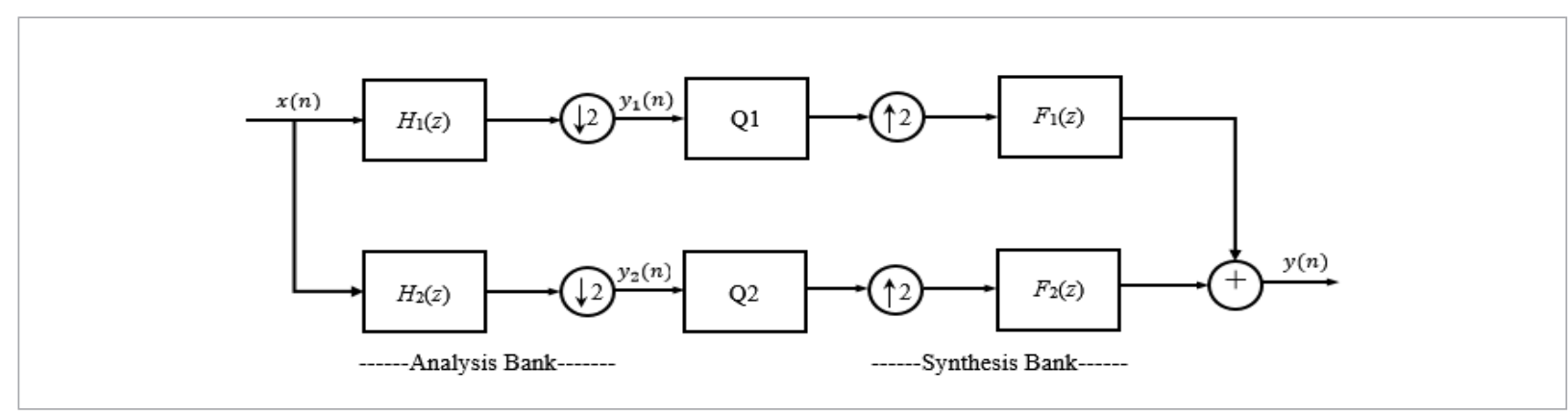


PCM is a commonly used method for obtaining digital representation of an analog signal in waveform coding. According to the G.711 coding standard, compression factor is equal to 255 . As we perform audio signal coding, we assume various compression factors for which we analyze the performance of the proposed coding scheme. In addition, we obtain the results of coding the same inputs with the implementation of quasi-logarithmic $\mu$-law PCM quantizer, for the purpose of comparison. As the proposed SBC schemes implement the same quantizer, the experiment can show the benefits of implementing sub-band coding and adaptation technique. This paper also puts emphasis on determining the optimal support limit values and the most efficient bit allocation for the purpose of achieving higher signal compression.

The rest of this paper is organized as follows. Section 2 describes the design and implementation of the non-adaptive quasi-logarithmic SBC scheme. The design and application of the backward-adaptive SBC scheme is presented in Section 3. The numerical results obtained in this research and the performances of the proposed coding schemes are the topics addressed in Section 4. Finally, Section 5 delivers the conclusions.

\section{Design of the Sub-Band Coding Scheme with a Quasi-Logarithmic Companding Quantizer}

As was already mentioned, the SBC process typically starts with decomposition of the input signal. In the coding scheme that we propose in this paper (Fig.1), the input signal is firstly decomposed by using two digital FIR filters: digital FIR low-pass frequency (LF) filter, denoted by $H_{1}(z)$ and a digital FIR high-pass frequency (HF) filter, denoted by $H_{2}(z)$. Synthesis and analysis filters are defined by the following relations [1]:

$F_{1}(z)=H_{2}(-z)$ and $F_{2}(z)=-H_{1}(-z)$,
$H_{1}(z)=H_{2}(-z)$
$H_{2}(z)=1-z^{-1}$.

One of the simplest exact reconstruction filter banks uses the Haar basis, and it is defined as [14]: $h_{1}(n)=(1 / 2,1 / 2), H_{1}(f)=e^{-j \pi f} \cos (\pi f)$,

$h_{2}(n)=(1 / 2,-1 / 2), H_{2}(f)=j e^{-j \pi f} \sin (\pi f)$.

The outputs of these filters provide us with two different signals (sub-bands), which can be processed independently. By using Eqs. (4) and (5), the utilized filters can be modelled by the following approximations in the time domain:

$$
y_{1}(n)=\frac{x(n)+x(n+1)}{2}, n=1,2, \ldots, M,
$$

$$
y_{2}(n)=\frac{x(n)-x(n+1)}{2}, n=1,2, \ldots, M,
$$

where $x(n)$ and $x(n+1)$ represent the input signal samples, $y_{1}(n)$ and $y_{2}(n)$ denote the sub-bands obtained from the FIR filters, while $M$ denotes the total number of input samples. After the input signal is processed by these filters, two independent signals, $y_{1}(n)$ and $y_{2}(n)$, with distinctly different statistical characteristics are obtained. With our proposal, the sub-bands are fed to the input of quasi-logarithmic companding quantizers, denoted by Q1 and Q2, designed for different bit rates. The advantage of the SBC scheme is that we can adapt quantization to the characteristics of the sub-bands, achieving higher output signal quality, compared to the widely used PCM scheme.

In companding quantization, the signal is firstly fed to a compressor, where it is compressed by applying a compressor function. Afterwards, the signal is fed to the uniform a quantizer, and finally, it is expanded by the expandor [6]. Since quasi-logarithmic quantizers are applied, the compressor uses $\mu$-law compressor function $[6,16]$ :

$$
c_{\mu}(x)=\frac{x_{\max }}{\ln (1+\mu)} \ln \left(1+\mu \frac{|x|}{x_{\max }}\right) \operatorname{sgn}(x),|x| \leq x_{\max },
$$

where $\mu$ represents the compression factor, while $x_{\max }$ denotes the support limit of the quantizer.

During the quantization process, an irreversible error is made due to rounding the current values of the input signal to a certain representation level. This error is called quantization error and it can be expressed by distortion, which is commonly defined as the average value of a mean-squared error [6]. For a symmetric 
probability density function, with infinite support region, distortion $D$ presents the sum of granular and overload distortions, $D=D_{\mathrm{g}}+D_{\mathrm{o}}$. In this research, an audio signal is modelled with a memoryless Laplacian source. It has been shown that the Laplacian source can be successfully used to model speech or audio signal [6]. Granular and overload distortion for quasi-logarithmic quantizer designed for Laplacian source of variance $\sigma^{2}$ and mean value equal to zero, can be calculated from $[2,12]$ :

$$
\begin{aligned}
& D_{\mathrm{g}}=\frac{\ln ^{2}(1+\mu) \sigma^{2}}{3 N^{2}}\left[\frac{1}{\mu^{2}} \frac{x_{\max }^{2}}{\sigma^{2}}+\frac{x_{\max }}{\sigma} \frac{\sqrt{2}}{\mu}+1\right], \\
& D_{\mathrm{o}}=\sigma^{2} \exp \left\{-\frac{\sqrt{2} x_{\max }}{\sigma}\right\} .
\end{aligned}
$$

It is well known that the determination of the support limit has a great impact on the overall signal distortion $[2,7,10]$. Greater support limit contributes to a bigger granular and smaller overload distortion, and vice versa $[2,7,10]$. The support limits in the non-adaptive quantization scheme are determined based on the experience from the previous research and by empirical investigation, so that $x_{\max 1}=10$ and $x_{\max 2}=3$ [12]. As mentioned, an objective quality measure used in the analysis is SQNR, defined as [6]:

$$
\operatorname{SQNR}[\mathrm{dB}]=10 \log _{10}\left(\frac{\sigma^{2}}{D}\right) \text {. }
$$

\section{A Sub-Band Coding Scheme with the Backward-Adaptive Quasi- Logarithmic Companding Quantizer}

The block scheme presented in Fig. 1 also corresponds to the backward adaptive coding scheme. In this case, Q1 and Q2 denote the backward adaptive quasi-logarithmic companding quantizers. The quantizers are fed with the signals of the sub-bands, as described in the previous section. This implies the necessity of defining the two support limit values and determining the variances of the sub-bands. The support limits are calculated for each frame, based on the previously quantized frame, so that they are adapted to the statistical characteristics of the signal. The problem occurs in the case of the first frame, since there is no frame before it to obtain the support limit value from and to start the iteration. In this case, the support limit values are designed for the fixed quantizer and Laplacian source. The values of the support limits for the first frame are determined as $x_{\max 1}^{\mathrm{BA}(1)}=10$ and $x_{\max 2}^{\mathrm{BA}(1)}=3$ [12]. The optimal support limit for the Laplacian source of unit variance is given as [10]:

$x_{\max }^{\mathrm{opt}}=\frac{1}{\sqrt{2}} \ln \left(\frac{3 \mu N^{2}}{\ln ^{2}(\mu+1)}\right)$,

where $N$ denotes the number of quantization levels.

The variances of the current, $i^{\text {th }}$ frame of the subbands samples, $\sigma_{1}^{2(i)}$ and $\sigma_{2}^{2(i)}$, can be calculated by using the following equations [6]:

$$
\sigma_{1}^{2(i)}=\frac{\sigma_{x}^{2(i)}}{2}(1+\rho)
$$

$$
\sigma_{2}^{2(i)}=\frac{\sigma_{x}^{2(i)}}{2}(1-\rho)
$$

where $\sigma_{x}^{2(i)}$ denotes the variance of the current frame of input signal samples. Variance can be calculated as:

$$
\sigma_{x}^{2(i)}=\frac{1}{L_{f}} \sum_{r=1}^{L_{f}} x(r)^{2},
$$

where $L_{f}$ represents the length of the frame, while $\rho$ represents the correlation coefficient of the input signal, determined as:

$$
\rho=\frac{\sum_{n=1}^{M-1} x(n) \times x(n+1)}{\sum_{n=1}^{M} x^{2}(n)} .
$$

It is assumed that the correlation factor does not change drastically through the frames, which have contributed to the choice of performing adaptation based on the variance. The adaptive support limit values, $x_{\max 1}^{\mathrm{BA}(i)}$ and $x_{\max 2}^{\mathrm{BA}(i)}$, are calculated for each frame individually, as follows. The standard deviations of the signals in the branches calculated for the previous 
Table 1

Variance, number of samples, amplitude dynamics and correlation coefficient of the input audio signal

\begin{tabular}{c|c|c|c|c}
\hline Input signal & Samples number & Signal variance & Correlation coefficient & Amplitude dynamics [dB] \\
\hline Classical music & 832531 & 0.0089 & 0.9938 & 90.0529 \\
\hline Rock music & 2741355 & 0.0361 & 0.9776 & 96.32 \\
\hline
\end{tabular}

frame are multiplied by the optimal value of the support limit for the Laplacian source of unit variance determined by Eq. (12):

$$
\begin{aligned}
& x_{\max 1}^{\mathrm{BA}(i)}=x_{\max }^{\mathrm{opt}} \sigma_{1}^{(i-1)}, i=2,3, \ldots, P, \\
& x_{\max 2}^{\mathrm{BA}(i)}=x_{\max }^{\mathrm{opt}} \sigma_{2}^{(i-1)}, i=2,3, \ldots, P,
\end{aligned}
$$

where $P$ represents the total number of frames. These steps represent an iterative process, by which we determine the adaptive support limits for each of the frames. This process represents the application of the backward adaptation.

To ensure the best possible quality of quantization, the bit allocation must be precisely determined. If the number of the sub-bands amounts to $L$ and the total bit rate available is denoted by $R$, the bit rate used for quantization of the particular sub-band can be calculated as [13]:

$$
R_{k}=R+\frac{1}{2} \log _{2} \frac{\sigma_{k}^{2}}{\prod_{k=1}^{L}\left(\sigma_{k}^{2}\right)^{\frac{1}{L}}},
$$

where $k$ denotes the ordinal number of the sub-band. The proposed scheme utilizes two sub-bands with the bit rates denoted by $R_{1}$ and $R_{2}$, which can be theoretically defined by implementing Eqs. (13) and (14) into Eq. (19):

$$
\begin{aligned}
& R_{1}=R+\frac{1}{4} \log _{2}\left(\frac{1+\rho}{1-\rho}\right), \\
& R_{2}=R+\frac{1}{4} \log _{2}\left(\frac{1-\rho}{1+\rho}\right) .
\end{aligned}
$$

\section{The Numerical Results and Analysis}

This section presents the performance analysis of the proposed SBC schemes. For this purpose, an experiment was conducted. We analyze the performance of the proposed coding schemes by using real audio signals as input. Additionally, we present the important results, notes and observations from the research. Table 1 shows some statistical parameters of the input signals: the number of samples, variance, correlation coefficient and amplitude dynamics of the audio signals used in this experiment.

As mentioned, adaptive support limit values are calculated for each frame, enabling us to use the value suitable for the current frame. The average of the experimentally calculated adaptive support limit values, obtained for all frames of the input signals and both quantizers in adaptive coding scheme are presented in Table 2 , for the frame size equal to 40 samples. One can notice that the average support limit values differ to a great extent from the values utilized in the first frame $\left(x_{\max 1}^{\mathrm{BA}(1)}=10\right.$ and $\left.x_{\max 2}^{\mathrm{BA}(1)}=3\right)$. Additionally, the values differ to a great extent for two input signals due to the difference in statistical characteristics of the signals, for which the support limits are calculated.

Bit rate is one of the most important parameters in signal compression, since it significantly determines the signal quality. In this experiment, we have chosen

\section{Table 2}

The average values of support limits for adaptive quantizers with backward adaptation

\begin{tabular}{l|c|c}
\hline \multicolumn{1}{c|}{ Input signal } & Average mean no. 1 & Average mean no. 2 \\
\hline Classical music & 0.2786 & 0.0371 \\
\hline Rock music & 1.1944 & 0.1275 \\
\hline
\end{tabular}


the bit rate of 9 bits per sample. This bit rate is expected to provide high SQNR values, i.e. high objective signal quality. The advantage of SBC is that we can use different bit rates for different sub-bands. This enables us to use higher bit-rate for the sub-band which carries a greater amount of information. In the case of audio signals, the sub-band after the LF filter caries significant amount of information of the input signal so that we could predict that higher bit rate should be used for this sub-band. By replacing the value of $\rho$ from Table 1 in Eqs. (20) and (21), we obtain the theoretical values of the bit rates for both sub-bands. Since both signals have a similar correlation factor value, the results are the same when rounded up to the closest integer value. The theoretical bit rates that should be used for the total bit rate of 9 bits per sample are $R_{1}=11$ and $R_{2}=7$ bits per sample. As these are the theoretical values, we conducted a practical examination of the coding schemes performance for different bit rate combinations. The examination of the non-adaptive SBC scheme was conducted in [12] and it was concluded that the most appropriate bit rate combination for this coding scheme is $R_{1}=10$ and $R_{2}=8$ bits per sample. This deviates from the theoretically obtained combination, and it was experimentally confirmed in [12]. Figure 2 presents the SQNR characteristics of the backward-adaptive sub-band coding scheme for various bit rate combinations. We considered the theoretically obtained bit allocation, the one used in [12], as well as the case when bit rates of the sub-bands are equal. One can observe that the theoretically obtained combination provides higher SQNR values. In the case of the classical music signal, the gain in SQNR amounts from $3.6 \mathrm{~dB}$ for all compression factor values. This confirms that, in the case of BASBC, bit rate combination of $R_{1}=11$ and $R_{2}=7$ bits per sample provides the highest SQNR values of all tested combinations

Numerical results from the application of the proposed SBC schemes in audio signal coding are presented in Tables 3 and 4 . The results are compared to those obtained for coding the same audio signal by using $\mu$-law PCM coding scheme [5]. The experiment was conducted for the cases of using two different frame sizes of 40 and 80 samples and four different compression factors, $\mu=\{5,100,255,511\}$, while the total bit rate amounts to $R=9$ bits per sample. The bit rate combinations are described above, while the to- tal bit rate represents the arithmetic mean of the bit rate used for each of the sub-bands. The difference in the bit rates of the branches is equal to 2 bits per sample, for the case of non-adaptive SBC, since it has been shown that this difference in bit rates outputs the highest SQNR values [12]. In the case of BASBC, the difference in bit rates of the branches amounts to 4 bits per sample, as presented in Fig. 2. Table 3 presents the performance of both SBC schemes as for the $\mu$-law PCM. It should be mentioned that the frame per frame processing applies only to backward adaptive SBC, while the non-adaptive SBC and $\mu$-law PCM do not implement division of the input signal into frames. The experiment was conducted for the case of using the given compression factor $\mu$, and the bit rates $R$ in all of the coding schemes compared.

\section{Figure 2}

SQNR characteristics of BASBC for different bit rate combinations and compression factors for classical music signal

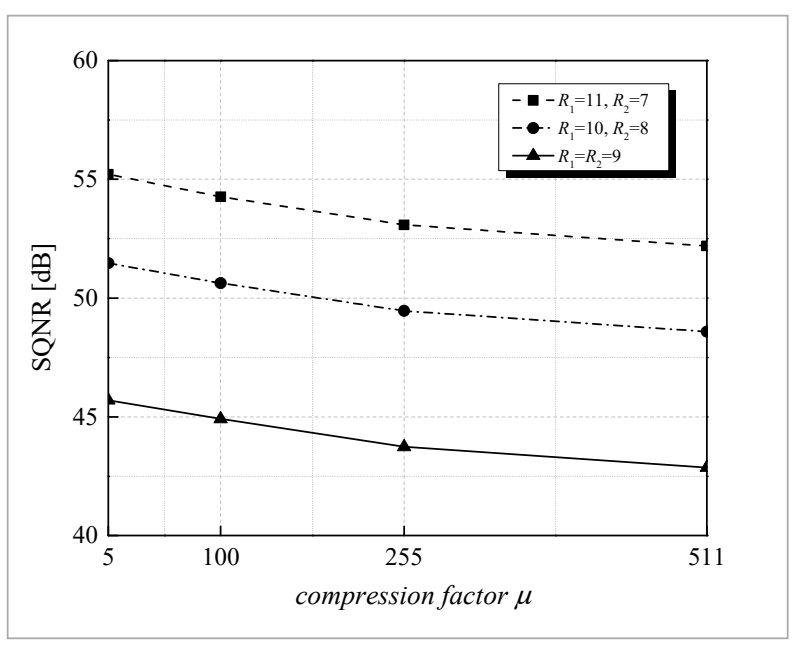

Table 3 shows that the implementation of the SBC scheme with backward-adaptive quantization (BAS$\mathrm{BC}$ ) provides the gain in SQNR for all values of the compression factor used, compared to the case of implementing the SBC scheme with the fixed quantizers. The results also present a significant gain in SQNR, when compared to the $\mu$-law PCM, ranging from around $10 \mathrm{~dB}$ up to $28.25 \mathrm{~dB}$. In the case when $R=9$ bits per sample and $\mu=5$, the SBC scheme with backward adaptive quantization outputs $\mathrm{SQNR}_{\mathrm{BASBC}}=55.2136 \mathrm{~dB}$ (when the frame size is 40), 
while SBC scheme with the fixed quantizers and $\mu$-law PCM scheme output $\mathrm{SQNR}_{\mathrm{SBC}}=28.5767 \mathrm{~dB}$ and $\mathrm{SQNR}_{\mathrm{PCM}}=26.9639 \mathrm{~dB}$, respectively. This confirms that the backward-adaptive SBC provides the gain in SQNR of about $26.6 \mathrm{~dB}$, when compared to non- adaptive SBC, while the gain amounts to about $28.25 \mathrm{~dB}$, compared to $\mu$-law PCM. The gain in SQNR decreases by increasing the compression factor value so that for compression factor $\mu=511$ and frame size equal to 40 samples, we have that $\mathrm{SQNR}_{\mathrm{BASBC}}=52.1915 \mathrm{~dB}$ and $\mathrm{SQNR}_{\mathrm{SBC}}=47.2552 \mathrm{~dB}$. In this case, one can ascertain the gain in SQNR of about $4.9 \mathrm{~dB}$, in favor of BASBC. For larger compression factor values, the gain in SQNR over PCM amounts to about $5.5 \mathrm{~dB}$ and 10.4 dB, when implementing non-adaptive and backward-adaptive SBC, respectively. The results are sim- ilar for the second frame size observed, and they are also presented in Table 3.

An overview of the results presented in Table 4 shows that both SBC schemes provide significant gain when compared to $\mu$-law PCM $\left(\mathrm{SQNR}_{\mathrm{PCM}}\right)$. As for the classical music input signal, backward adaptive SBC provides the gain in SQNR compared to the other coding schemes considered in the analysis. The gain is especially noticeable when using lower compression factor values as the adaptation has a major impact on the objective quality of the output, providing significant gain in SQNR. When a rock musing signal is on the input and compression factor $\mu$ is equal to 5 , the gain in SQNR amounts to around $16 \mathrm{~dB}$, compared to non-adaptive $\mathrm{SBC}$ and up to about $18 \mathrm{~dB}$, compared to $\mu$-law PCM (see Table 4). One can notice that the ap-

Table 3

SQNR for different frame sizes and compression factors obtained for classical music signal

\begin{tabular}{|c|c|c|c|c|c|}
\hline $\begin{array}{c}\text { Bit rate } \\
\text { [bits per sample] }\end{array}$ & $\begin{array}{l}\text { Frame size } \\
\text { [samples] }\end{array}$ & Compression factor $\mu$ & $\begin{array}{l}\mathbf{S Q N R}_{\text {BASBC }} \\
{[\mathrm{dB}]}\end{array}$ & $\begin{array}{l}\mathrm{SQNR}_{\mathrm{SBC}} \\
{[\mathrm{dB}]}\end{array}$ & $\begin{array}{l}\mathrm{SQNR}_{\mathrm{PCM}} \\
{[\mathrm{dB}]}\end{array}$ \\
\hline \multirow{8}{*}{9} & \multirow{4}{*}{40} & 5 & 55.2136 & 28.5767 & 26.9639 \\
\hline & & 100 & 54.2638 & 44.1071 & 40.0344 \\
\hline & & 255 & 53.0825 & 46.6549 & 41.5870 \\
\hline & & 511 & 52.1915 & 47.2552 & 41.7694 \\
\hline & \multirow{4}{*}{80} & 5 & 54.5946 & 28.5767 & 26.9639 \\
\hline & & 100 & 54.4935 & 44.1071 & 40.0344 \\
\hline & & 255 & 53.3451 & 46.6549 & 41.5870 \\
\hline & & 511 & 52.4748 & 47.2552 & 41.7694 \\
\hline
\end{tabular}

Table 4

SQNR for different frame sizes and compression factors obtained for rock music signal

\begin{tabular}{|c|c|c|c|c|c|}
\hline $\begin{array}{c}\text { Bit rate } \\
\text { [bits per sample] }\end{array}$ & $\begin{array}{c}\text { Frame size } \\
\text { [samples] }\end{array}$ & Compression factor $\mu$ & $\begin{array}{l}\mathbf{S Q N R}_{\mathrm{BASBC}} \\
{[\mathrm{dB}]}\end{array}$ & $\begin{array}{l}\mathbf{S Q N R}_{\mathrm{SBC}} \\
{[\mathrm{dB}]}\end{array}$ & $\begin{array}{l}\mathbf{S Q N R}_{\mathrm{PCM}} \\
{[\mathrm{dB}]}\end{array}$ \\
\hline \multirow{8}{*}{9} & \multirow{4}{*}{40} & 5 & 50.6572 & 34.6374 & 32.4916 \\
\hline & & 100 & 49.9723 & 46.9182 & 42.5586 \\
\hline & & 255 & 48.8659 & 47.7685 & 42.7975 \\
\hline & & 511 & 48.0651 & 47.5891 & 42.4244 \\
\hline & \multirow{4}{*}{80} & 5 & 50.6080 & 34.6374 & 32.4916 \\
\hline & & 100 & 50.3481 & 46.9182 & 42.5586 \\
\hline & & 255 & 49.2487 & 47.7685 & 42.7975 \\
\hline & & 511 & 48.4233 & 47.5891 & 42.4244 \\
\hline
\end{tabular}


plication of the backward adaptive quantization enables using significantly smaller compression factor values, while performance of the non-adaptive SBC improves with using larger compression factor values (e.g. $\mu=511$ ). In the case of rock music signal, when the frame size is equal to 40 and compression factor amounts to 511, BASBC provides the gain in SQNR of about $0.47 \mathrm{~dB}$ in comparison to non-adaptive $\mathrm{SBC}$ and about 5.6 dB when compared to $\mu$-law PCM. The situation is similar for using larger frame sizes, and the results are presented in the tables.

The performance of a coding scheme can also be observed through SQNR characteristics determined for different bit rates. The following figures present SQNR characteristics of the proposed BASBC scheme compared to SBC and $\mu$-law PCM system, for two compression factors of $\mu=5$ and $\mu=255$, while the classical music signal in on the input. We have chosen these specific values since they reflect the implementation of small and large compression factor values, respectively. SQNR characteristics present the objective quality of the considered coding schemes in the wide range of bit rates, which can be used in high quality audio signal coding.

The figures clearly show the importance of implementing backward adaptation in BASBC, when smaller values of compression factor are used. Moreover, Fig. 4 shows that BASBC also provides gain when larger compression factor is implemented, for all bit

\section{Figure 3}

SQNR characteristics of the proposed BASBC scheme compared to SBC and PCM, for compression factor of $\mu=5$

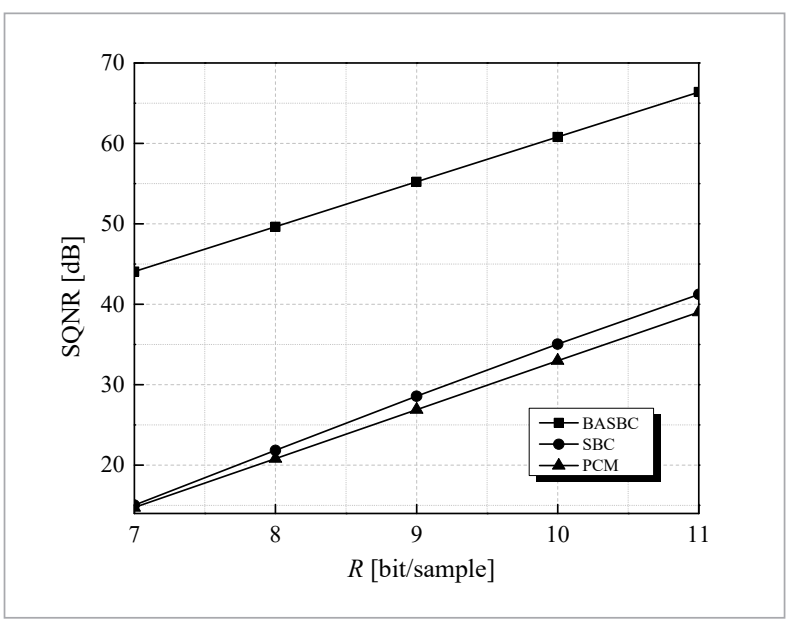

\section{Figure 4}

SQNR characteristics of the proposed BASBC, SBC and PCM scheme, for compression factor $\mu=255$

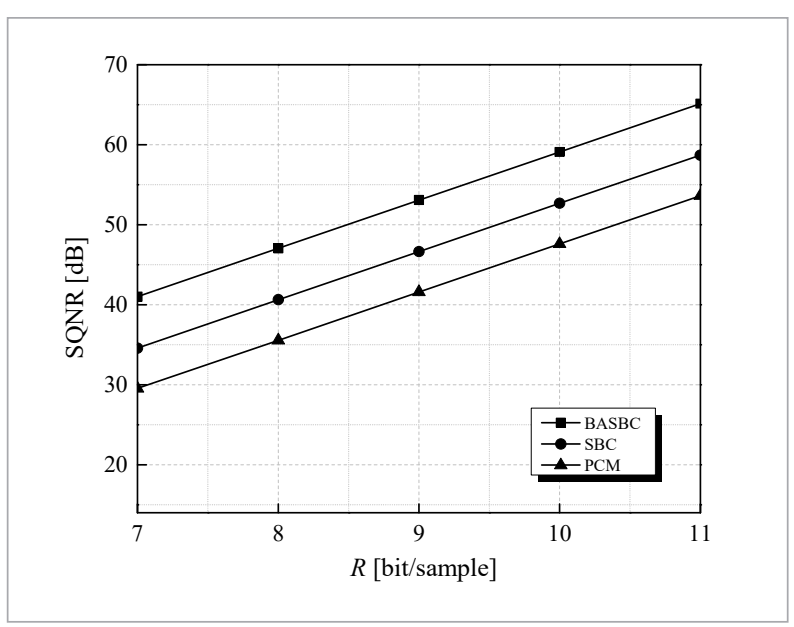

rates used in the experiment. As one can notice from Figs. 3 and 4, BASBC and non-adaptive SBC provide constant gain in SQNR when compared to $\mu$-law PCM, while BASBC scheme provides gain in SQNR, compared to the non-adaptive SBC and $\mu$-law PCM, for all bit rates.

\section{Conclusions}

This paper has presented the design of the non-adaptive and backward adaptive SBC scheme, with the implementation of quasi-logarithmic Laplacian source quantization. The proposed schemes have been implemented in processing a real audio signal. We have demonstrated the possible choice for the bit allocation used in the sub-bands, and presented the results for the cases of using various compression factors and frame sizes. We have also shown that both SBC schemes have their advantages over the $\mu$-law PCM system, providing noticeable higher SQNR values. Moreover, we have observed that backward-adaptive SBC is especially convenient for using smaller compression factor values. In this case, we have ascertained significantly higher SQNR values compared to the ones of non-adaptive SBC coding scheme and the $\mu$-law PCM system. The coding schemes, presented in this paper, demonstrate the implementation of a simple sub-band coding and quantization techniques in audio signal coding. It has been shown that with ap- 
propriate design and parameter choice, this quantizer provides high performance with relative low computational complexity. Eventually, we can anticipate that the proposed coding schemes have great possibilities for improvement, with the application of the different digital FIR filters and quantization techniques,

\section{References}

1. Agrawal, S., Sahu, O. Polyphase Structure Based Eigen Design of Two-Channel Quadrature Mirror Filter Bank. International Journal of Image, Graphics and Signal Processing (IJIGSP), 2014, 6(10), 29-35. https://doi. org/10.5815/ijigsp.2014.10.04

2. Aleksić, D., Perić, Z., Nikolić, J. Support Region Determination of the Quasilogarithmic Quantizer for Laplacian Source. Przeglad Elektrotechniczny, 2012, 88(7A), 130-132. Available at http://red.pe.org.pl/articles/2012/7a/28.pdf

3. Aziz, A. Subband Coding of Speech Signals Using Decimation and Interpolation. 13th International Conference on Aerospace Sciences and Aviation Technology, ASAT-13, Cairo (Egypt), 2009, 26-28.

4. Gharavi, H., Tabatabai, A. Sub-band Coding of Monochrome and Color Images. IEEE Transactions on Circuits and Systems, 1988, 35(2), 207-214. https://doi. org/10.1109/31.1723

5. ITU-T, Recommendation G.711. Pulse Code Modulation (PCM) of Voice Frequencies. International Telecommunication Union, 1988.

6. Jayant, N. S., Noll, P. Digital Coding of Waveforms. New Jersey, Prentice Hall, Chapter 5, 221-251, 1984.

7. Na, S. On the Support of Fixed-Rate Minimum MeanSquared Error Scalar Quantizers for a Laplacian Source. IEEE Transactions on Information Theory, 2004, 50(5), 937-944. https://doi.org/10.1109/TIT.2004.826686

8. Na, S. Neuhoff, D. L. On the Support of MSE-Optimal, Fixed-Rate, Scalar Quantizers. IEEE Transactions on Information Theory, 2001, 47(7), 2972-2982. https:// doi.org/10.1109/18.959274

9. Naik, L. R., Devaraja Naik, R. L. Sub-band Coding of which is left to the future research.

\section{Acknowledgments}

This work is supported by Serbian Ministry of Education, Science and Technologic Development (Project TR 32035).

Noisy Speech Signals Using Digital Signal Processing. International Journal of Advanced Research in Electronics and Communication Engineering (IJARECE), 2015, 4(4), 901-904.

10. Perić, Z., Aleksić, D., Stefanović, M., Nikolić, J. New Approach to Support Region Determination of the $\mu$-law Quantizer. Electronics and Electrical Engineering, 2013, 19(8), 111-114. https://doi.org/10.5755/j01.eee.19.8.5406

11. Perić, Z., Nikolić, J., Mosić, A., Panić, S. A Switched Adaptive Quantization Technique Using $\mu$-law Quantizers. Information Technology and Control, 2010, 39(4), 317-320. Available at http://www.itc.ktu.lt/index. php/ITC/article/view/12388

12. Peric, Z., Tancic, M., Tomic, S., Ciric, D. Subband Coding of Audio Signal with Logarithmic Compandors. 12th International Conference on Telecommunication in Modern Satellite, Cable and Broadcasting Services (TELSIKS). https://doi.org/10.1109/TELSKS.2015.7357728

13. Sayood, K. Introduction to Data Compression. Elsevier Inc., 3rd ed, 2005.

14. Tan, S., Fischer, T. Linear Prediction of Subband Signals. IEEE Journal on Selected Areas in Communications, 1994, 12(9), 1576-1583. https://doi. org/10.1109/49.339926

15. Taubman, D., Zakhor, A. Multirate 3-D Subband Coding of Video. IEEE Transactions on Image Processing, 1994, 3(5), 572-588. https://doi.org/10.1109/83.334984

16. Thang, P. Four-channel Sub-band Coding with Combination of [8 84 2] for Digital Audio Signal Processing. Science Journal of Circuits, Systems and Signal Processing, 2013, 2(5), 106-111. https://doi.org/10.11648/j. cssp.20130205.12 